\title{
An unusual intermolecular interaction between a lone pair and an electron-rich $\pi$-electron system of a quinoid dianion
}

\author{
Supporting Information
}

Vedran Vuković, ${ }^{a}$ Tomislav Piteša ${ }^{\mathrm{b}}$, Christian Jelsch, ${ }^{\mathrm{a}}$ Emmanuel Wenger, ${ }^{a}$ Krešimir Molčanov*b

${ }^{\text {a }}$ CRM2, CNRS, Université de Lorraine, 54000 Nancy, France

${ }^{\mathrm{b}}$ Ruđer Bošković Institute, Bijenička 54, HR-10000 Zagreb, Croatia

e-mail: kmolcano@irb.hr

\author{
S1 Analysis of residual density \\ S2 Atomic charges and electrostatic potential \\ S3 Additional details on molecular geometry and charge density \\ S4 Comparison of experimental and optimised contact between DHQ and DMSO \\ S5 References
}

S1 Analysis of residual density

Residual density analysis after multipolar refinement allowed for assessment of refinement quality. Residual density descriptors were compared with values expected from experimental data with a purely Gaussian noise.

The largest minimum and maximum in experimental residual density maps are -0.44 and 0.46 e $\AA^{-3}$ respectively. This is acceptable for a compound containing a third-row element and with somewhat limited X-ray data resolution. The ratio of observed and calculated reflection intensities is nearly constant with respect to reciprocal resolution (figure S1). Electron density 
in the quinone ring plane is fairly uniform (figure S2). Fractal dimensions of different residual density iso-surfaces form an approximate parabola (figure S3). The distribution of standardised difference of experimental and theoretical structure factors is approximately normal (figure S4). Standard deviations of total density around the quinone ring are almost indistinguishable between theoretical and experimental models (figure S5). The refined model, hence, represents experimental data well.

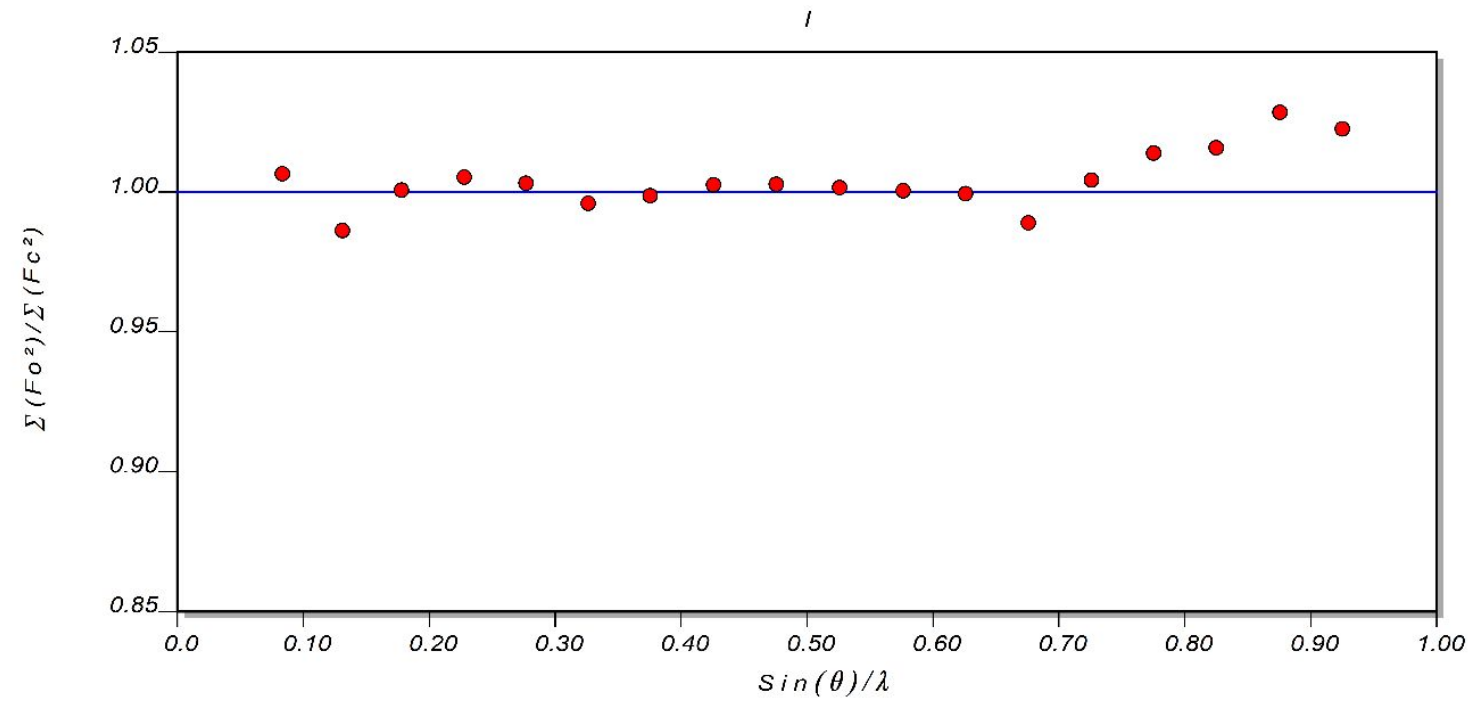

Figure S1 Average $I_{\mathrm{obs}} / I_{\text {calc }}$ ratio scaling with reciprocal resolution in LiDHQ.
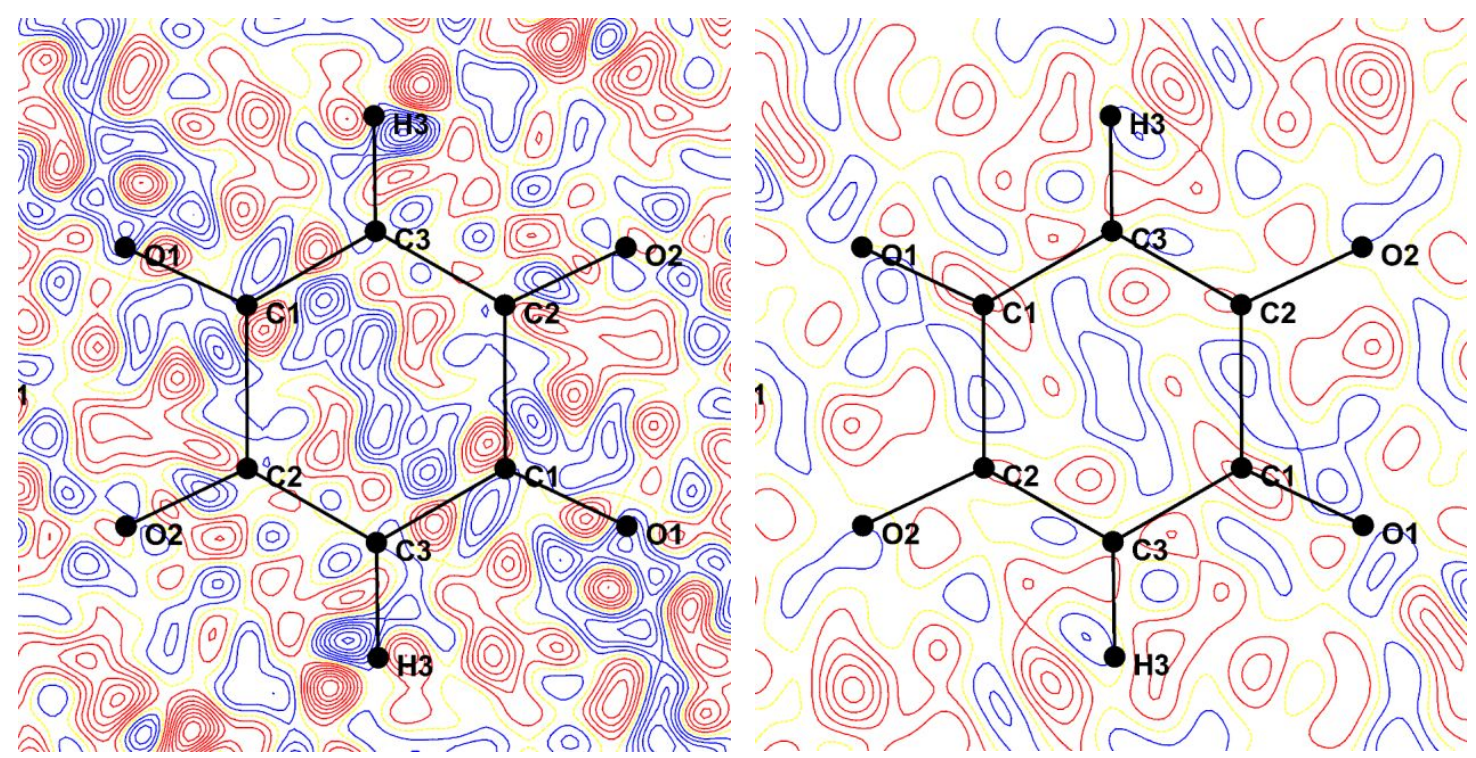

Figure S2 Residual electron density in quinone rings after multipolar refinement when including all reflections (left) or only those up to reciprocal resolution of $0.7 \AA^{-1}$ (right). Blue 
contours represent positive, while red represent negative residual density; the yellow line represents zero. The contour spacing is $0.05 \mathrm{e} \AA^{-1}$.

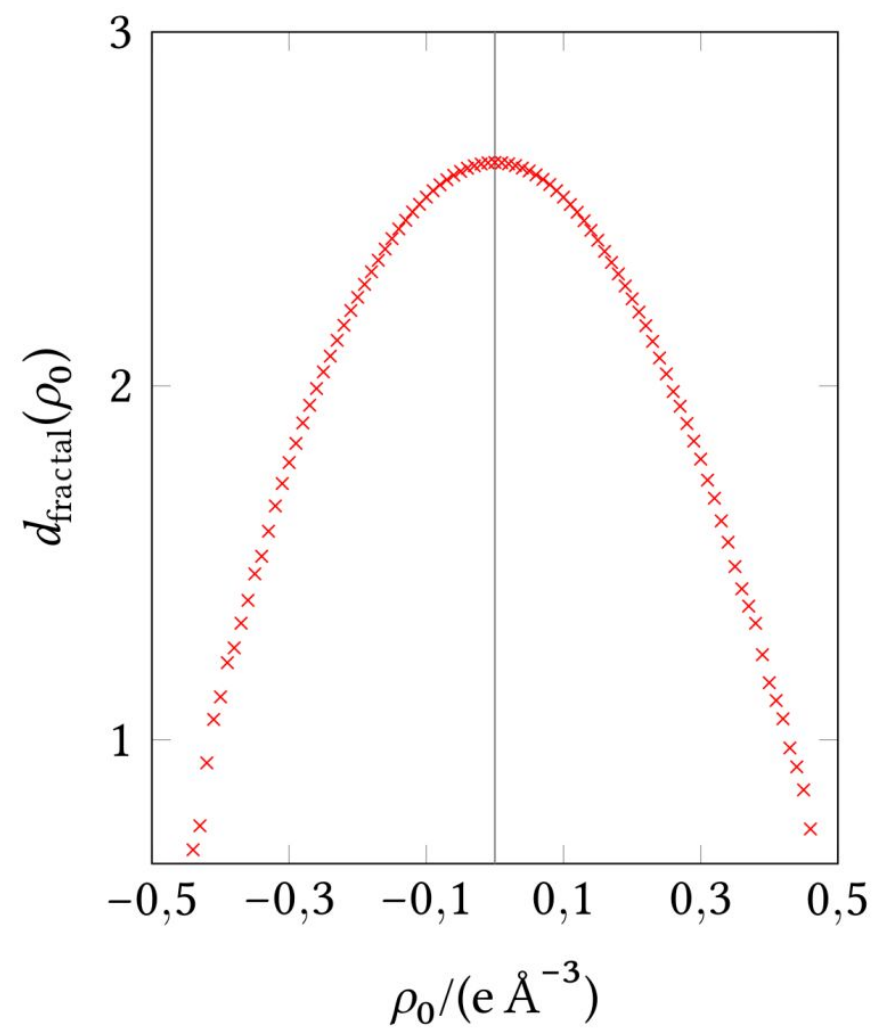

Figure S3 Dependence of isosurface fractal dimension of residual density on the residual density value in $\mathbf{1}$. 


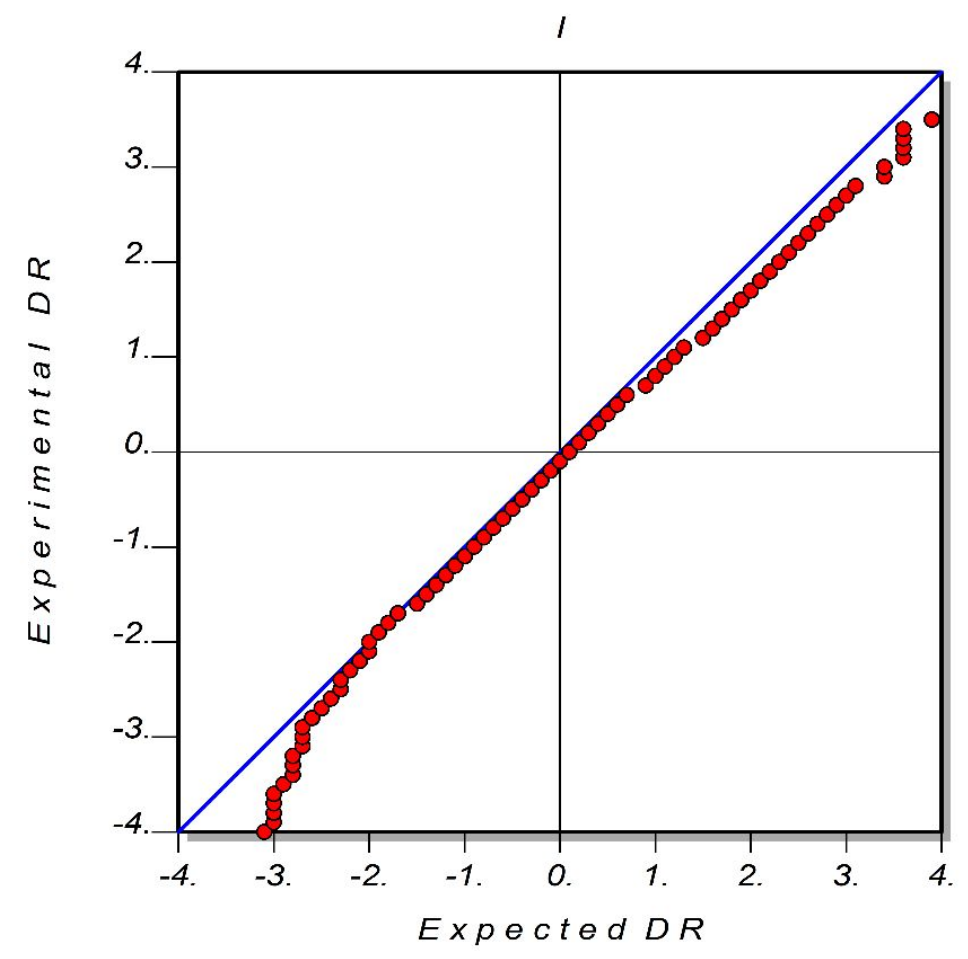

Figure S4 Quantile-quantile plot of experimental $\Delta R=\left(I_{\mathrm{obs}}-I_{\text {calc }}\right) /\left(1.03 \sigma\left(I_{\mathrm{obs}}\right)\right)$ with respect of theoretical quantile distribution under the assumption of normal distribution in $\mathbf{1}$.
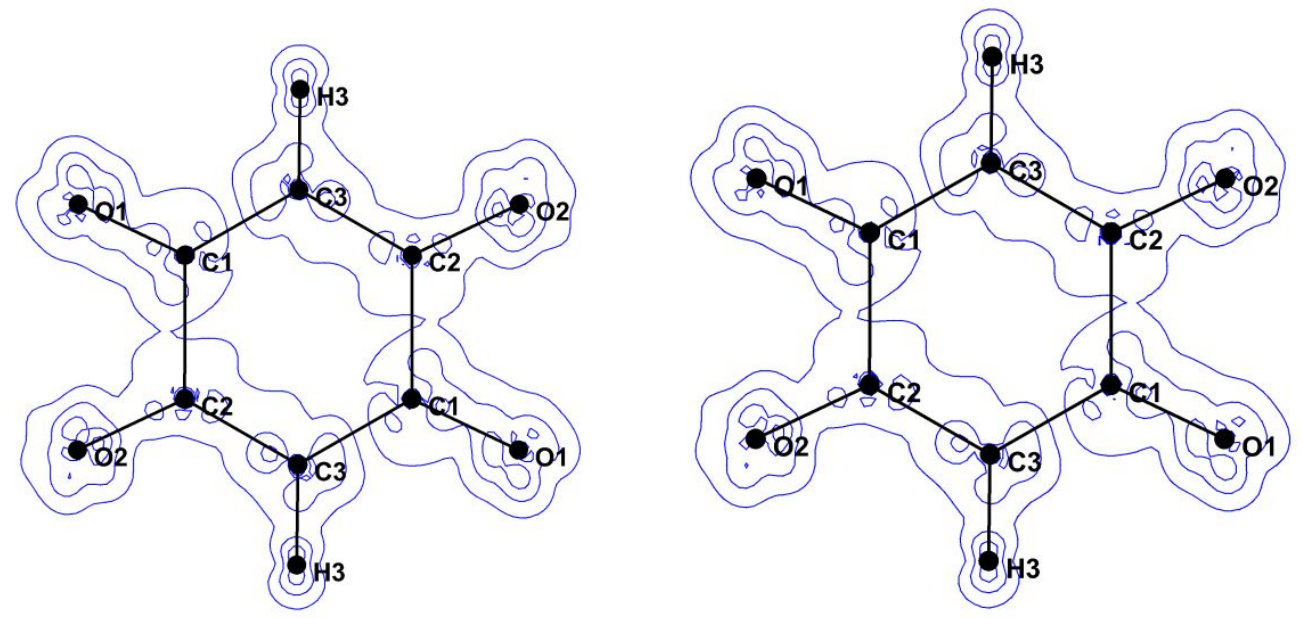

Figure S5 Total charge density standard deviations in the ring plane of DHQ dianion for the experimental (left) and theoretical (right) model. The contour level is $0.001 \mathrm{e} \AA^{-1}$. 


\section{S2 Atomic charges and electrostatic potential}

Atomic and molecular charges. The model $N_{\mathrm{val}}-P_{\mathrm{val}}$ and Bader charges for atoms in experimental charge density models are well-correlated (Table S2), except that the Bader charges are typically larger in absolute value. The total charges for water and DMSO are close to neutral, while $\mathrm{Li}^{+}$and $\mathrm{DHQ}^{2-}$ charges are close to +1 and -2 , respectively. The only major difference is in $\mathrm{C} 1$ and $\mathrm{C} 2$ atoms of $\mathrm{DHQ}^{2-}$ : they are significantly positively charged according to Bader partitioning, while almost uncharged according to the $P_{\mathrm{val}}$-derived charges.

Theoretical charge density models are not as well-correlated as experimental ones (Table S2). The signs of many charges are inversed, most notably in $q_{\text {model }}$ from water and DMSO methyl groups. Bader charges reflect experimental values somewhat better.

Table S1 Comparison of $N_{\mathrm{val}}-P_{\mathrm{val}}$ atomic charges (e) derived from experimental $P_{\mathrm{val}}$ in the quinoid dianions DHQ, CA [1] and NA [2].

\begin{tabular}{llll}
\hline & DHQ & CA & NA \\
\hline O1 & -0.44 & $-0.28(2)$ & $-0.24(3)$ \\
O2 & -0.45 & $-0.28(2)$ & $-0.29(3)$ \\
$\mathrm{C} 1$ & 0.00 & $-0.03(3)$ & $-0.02(4)$ \\
$\mathrm{C} 2$ & $-0.03(3)$ & $-0.04(4)$ \\
$\mathrm{C} 3$ & +0.01 & $+0.05(5)$ & $-0.04(7)$ \\
\hline substituent & -0.24 & $\mathrm{Cl}$ & $\mathrm{NO}_{2}$ \\
& $\mathrm{H}$ & $-0.36(3)$ & -0.36 \\
\hline total anion & +0.17 & -1.86 & -0.96 \\
\hline
\end{tabular}


Table S2 Model and topological charges (e) of atoms and molecules in $\mathbf{1 .}$

\begin{tabular}{|c|c|c|c|c|}
\hline \multirow[b]{2}{*}{ Atom/group } & \multicolumn{2}{|c|}{$\left(N_{\mathrm{val}}-P_{\mathrm{val}}\right) / e$} & \multicolumn{2}{|l|}{$q_{\text {Bader }} / \mathrm{e}$} \\
\hline & exp. & theor. & exp. & theor. \\
\hline $\mathrm{C} 1$ & $-0.01(7)$ & $-0.07(2)$ & $0.65(2)$ & 0.74 \\
\hline $\mathrm{C} 2$ & $0.01(5)$ & $-0.03(2)$ & $0.67(2)$ & $0.63(1)$ \\
\hline $\mathrm{C} 3$ & $-0.23(4)$ & $0.15(2)$ & $-0.11(3)$ & $-0.04(1)$ \\
\hline $\mathrm{O} 1$ & $-0.45(2)$ & $-0.37(1)$ & $-1.13(2)$ & -1.02 \\
\hline $\mathrm{O} 2$ & $-0.44(2)$ & $-0.38(1)$ & $-1.11(1)$ & -0.96 \\
\hline $\mathrm{H} 3$ & $0.17(4)$ & $-0.09(1)$ & $0.11(1)$ & $-0.10(1)$ \\
\hline Li1 & $0.98(1)$ & 0.98 & 0.91 & 0.91 \\
\hline S1 & $0.41(7)$ & $0.09(1)$ & $1.26(3)$ & 1.05 \\
\hline $\mathrm{O} 3$ & $-0.67(4)$ & -0.50 & $-1.26(1)$ & -1.19 \\
\hline $\mathrm{C} 4$ & $-0.71(8)^{*}$ & $0.75(3)$ & $-0.35(3)$ & $0.06(1)$ \\
\hline $\mathrm{C} 5$ & $-0.71(8)^{*}$ & $0.79(2)$ & $-0.35(3)$ & $0.15(1)$ \\
\hline $\mathrm{H} 4 \mathrm{~A}$ & $0.28(2)^{*}$ & $-0.21(1)$ & $0.14(1)$ & -0.03 \\
\hline $\mathrm{H} 4 \mathrm{~B}$ & $0.28(2)^{*}$ & $-0.19(1)$ & $0.15(1)$ & 0.00 \\
\hline $\mathrm{H} 4 \mathrm{C}$ & $0.28(2)^{*}$ & $-0.23(1)$ & $0.15(1)$ & -0.06 \\
\hline $\mathrm{H} 5 \mathrm{~A}$ & $0.28(2)^{*}$ & $-0.17(1)$ & $0.14(1)$ & 0.01 \\
\hline $\mathrm{H} 5 \mathrm{~B}$ & $0.28(2)^{*}$ & $-0.18(1)$ & $0.15(1)$ & 0.01 \\
\hline $\mathrm{H} 5 \mathrm{C}$ & $0.28(2)^{*}$ & $-0.30(1)$ & $0.15(1)$ & $-0.17(1)$ \\
\hline $\mathrm{O} 1 \mathrm{~W}$ & $-0.36(1)^{* *}$ & $0.06(4)$ & -0.94 & $-1.12(1)$ \\
\hline H1WA & $0.17^{* *}$ & $-0.04(2)$ & 0.46 & 0.58 \\
\hline H1WB & $0.17^{* *}$ & $-0.05(3)$ & 0.46 & 0.52 \\
\hline $\mathrm{DHQ}^{2-}$ & $-1.90(8)$ & $-1.58(3)$ & $-1.83(4)$ & $-1.50(2)$ \\
\hline DMSO & $0.00(1)$ & -0.15 & 0.19 & -0.16 \\
\hline $\mathrm{H}_{2} \mathrm{O}$ & $-0.02(1)$ & $-0.03(5)$ & -0.02 & $-0.02(1)$ \\
\hline
\end{tabular}

* These values are the same because they were restrained to be equal during refinement. The corresponding values in the experimental Bader charge column are consequently heavily correlated.

** The charge density parameters for this water molecule are all taken from the ELMAM2 database [3] and restrained to database values during experimental model refinement.

Correlation coefficients: model exp./Bader exp. 79.79\%, model theor./Bader theor. $47.71 \%$, model exp./model theor. $26.19 \%$, Bader exp./Bader theor. 95.23\%.

Standard deviations smaller than the last decimal place cited are omitted. 


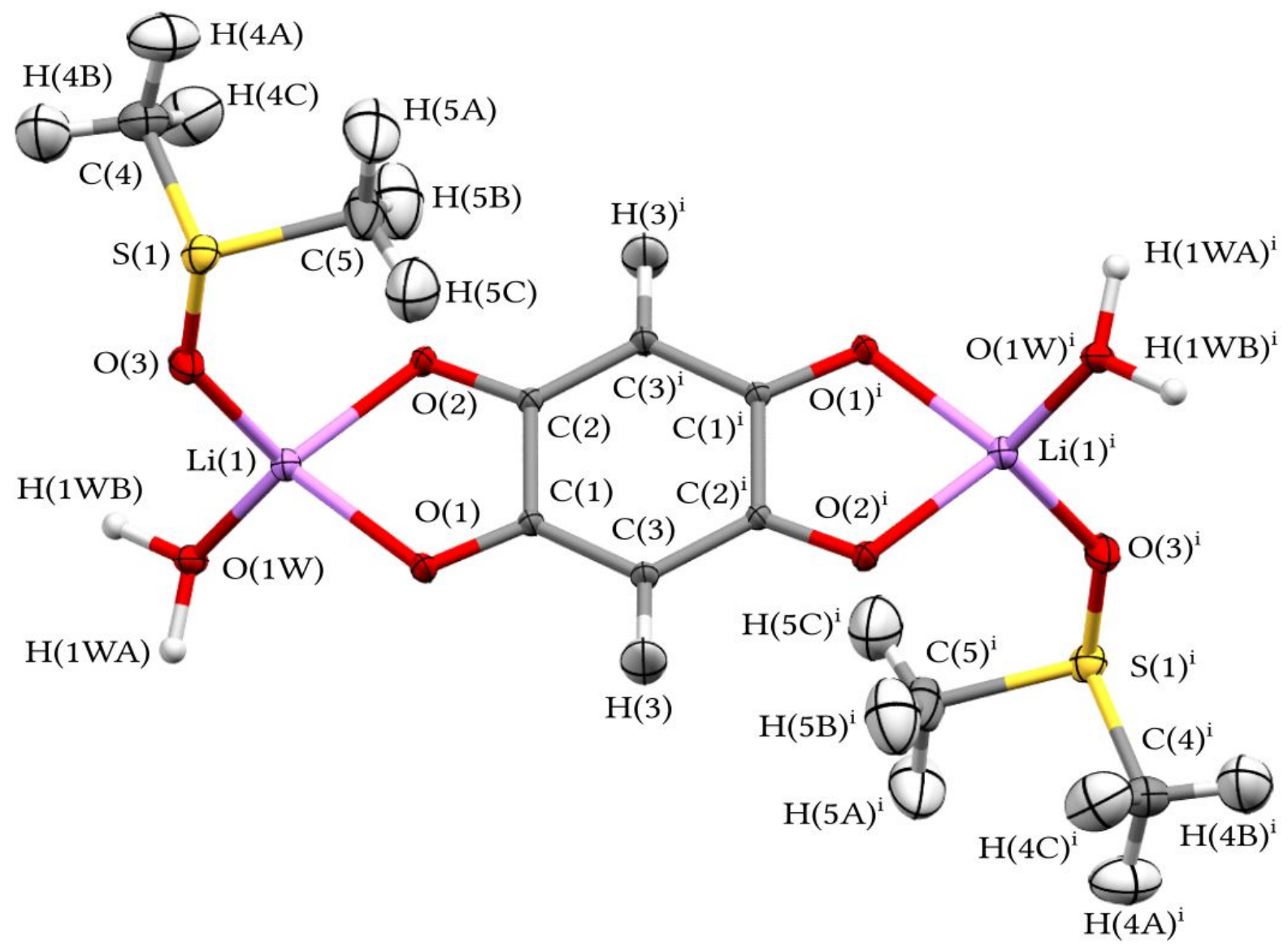

Figure S6 Displacement ellipsoid plot (50 \% probability) of $\mathrm{Li}_{2} \mathrm{DHQ}\left(\mathrm{H}_{2} \mathrm{O}\right)_{2}(\mathrm{DMSO})_{2}$ unit with atom numbering scheme. Symmetry operator $i)-x,-y,-z$. 

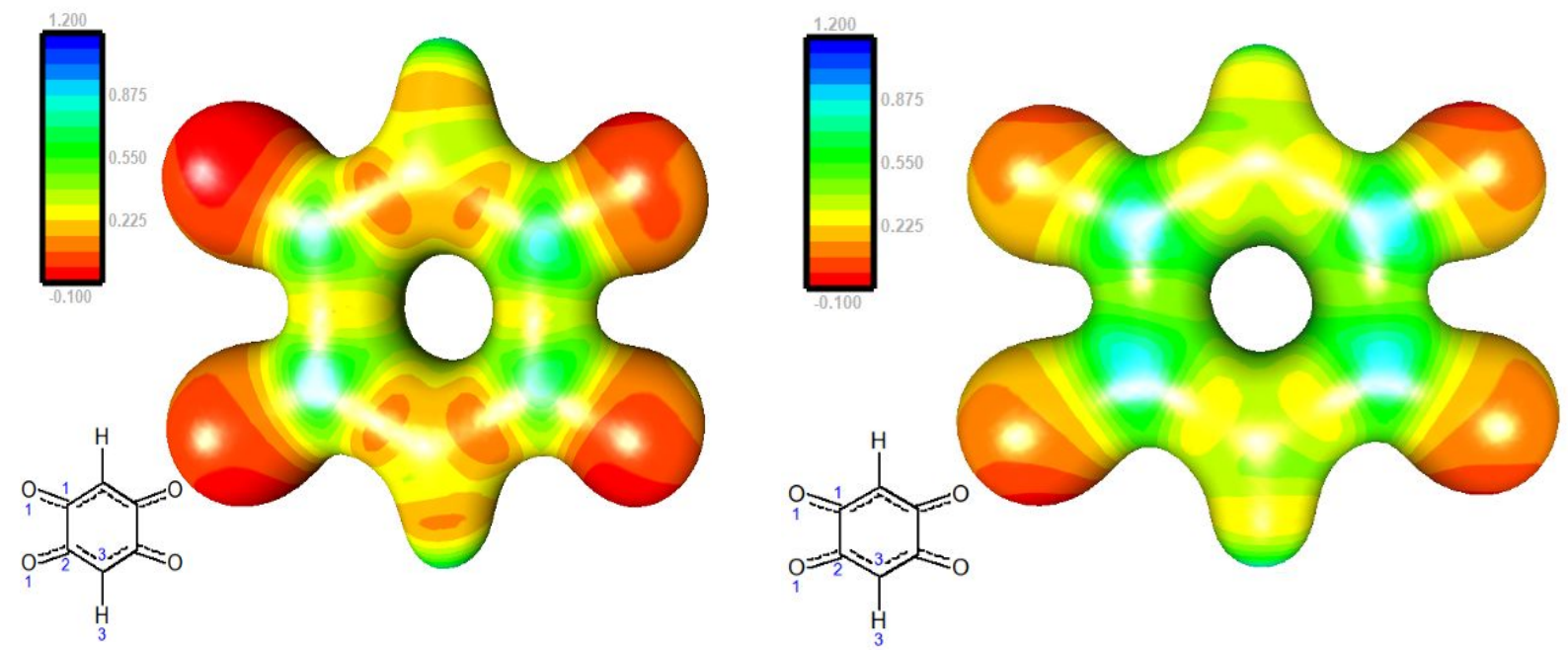

Figure S7 Electrostatic potential distribution from experimental (left) and theoretical (right) model of DHQ dianion charge density mapped onto $0.5 \mathrm{e} \AA^{-3}$ total electron density isosurface.
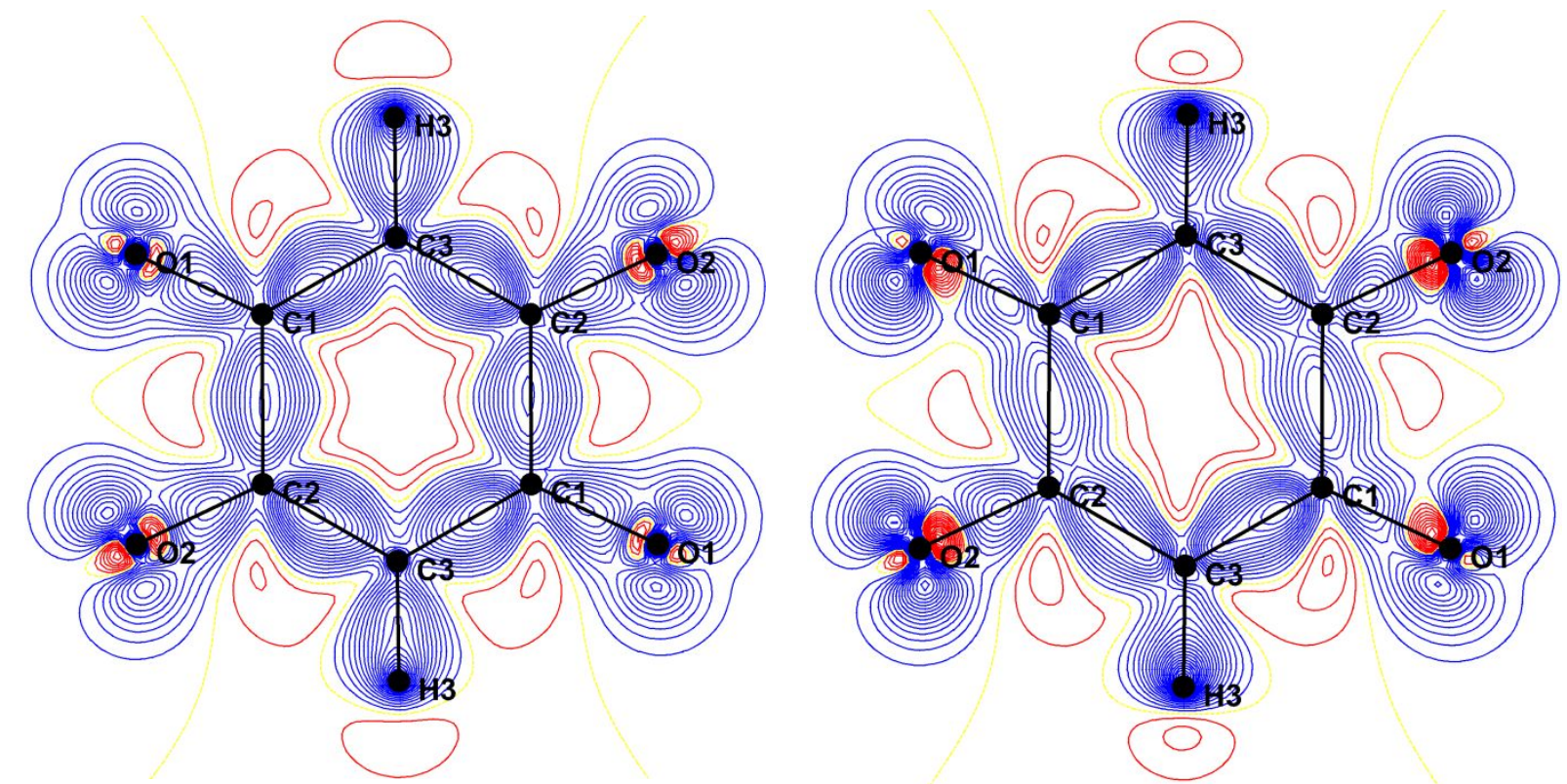

Figure S8 Deformation density in DHQ dianion after multipolar refinement of experimental (left) and theoretical (right) models. Blue colour represents positive, and red negative density; the yellow line represents zero. The spacing between two contours is $0.05 \mathrm{e} \AA^{-3}$. 


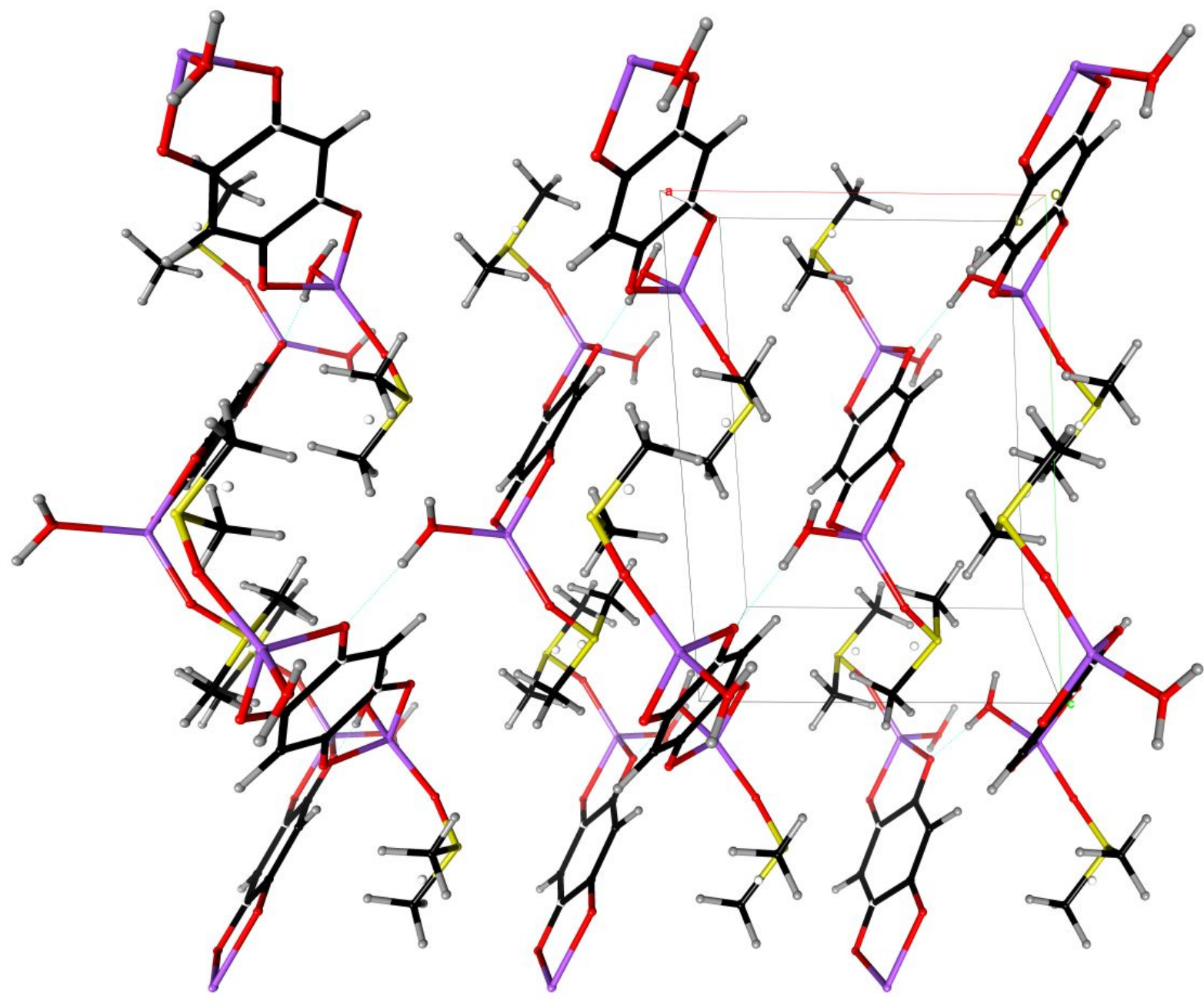

Figure S8 Crystallographic autostereogram of I viewed along the direction [010]. The H-O$\mathrm{H} \cdots \mathrm{O}=\mathrm{C}$ are shown in blue dotted lines.

Table S3 Topological parameters of chemical bonds in compound 1.

\begin{tabular}{|c|c|c|c|c|c|c|c|c|c|}
\hline \multirow[b]{2}{*}{ Bond } & \multirow[b]{2}{*}{ Length/Å } & \multicolumn{2}{|c|}{ Bond order } & \multicolumn{2}{|c|}{ Ellipticity } & \multicolumn{2}{|c|}{$\rho / \mathrm{e} \AA^{-3}$} & \multicolumn{2}{|c|}{$\nabla^{2} \rho /$ e $\AA^{-5}$} \\
\hline & & exp. & theor. & exp. & theor. & exp. & theor. & exp. & theor. \\
\hline $\mathrm{C} 1-\mathrm{C} 2$ & $1.5348(7)$ & $0.77(3)$ & $0.70(1)$ & $0.14(2)$ & 0.09 & $1.68(1)$ & 1.61 & $-11.0(4)$ & $-9.5(1)$ \\
\hline $\mathrm{C} 2-\mathrm{C} 3$ & $1.4004(7)$ & $1.45(4)$ & $1.19(1)$ & $0.25(2)$ & 0.29 & $2.14(1)$ & 2.08 & $-19.2(7)$ & $-16.0(2)$ \\
\hline
\end{tabular}




\begin{tabular}{|c|c|c|c|c|c|c|c|c|c|}
\hline $\mathrm{C} 3-\mathrm{C} 1^{\mathrm{i}}$ & $1.3965(7)$ & $1.41(4)$ & $1.12(1)$ & $0.32(2)$ & 0.27 & $2.10(1)$ & 1.99 & $-18.0(6)$ & $-14.3(3)$ \\
\hline $\mathrm{C} 1-\mathrm{O} 1$ & $1.2656(7)$ & $1.34(3)$ & $1.37(1)$ & $0.08(3)$ & 0.06 & $2.54(2)$ & $2.49(1)$ & $-23.3(28$ & $-24.7(7)$ \\
\hline & & & & & & & & ) & \\
\hline \multirow[t]{2}{*}{$\mathrm{C} 2-\mathrm{O} 2$} & $1.2630(6)$ & $1.30(2)$ & $1.30(1)$ & $0.05(2)$ & $0.05(1)$ & $2.50(3)$ & $2.40(1)$ & $-21.7(31$ & $-21.0(7)$ \\
\hline & & & & & & & & ) & \\
\hline $\mathrm{C} 3-\mathrm{H} 3$ & $1.083(8)$ & $0.63(4)$ & $0.74(1)$ & 0.07 & 0.06 & $1.81(1)$ & 1.80 & $-15.7(6)$ & $-14.7(3)$ \\
\hline O1-Li1 & $1.961(1)$ & $-*$ & $-*$ & 0.03 & 0.02 & 0.15 & 0.15 & 4.9 & 5.0 \\
\hline O2-Li1 & $1.972(1)$ & $-*$ & $-*$ & 0.02 & 0.02 & 0.16 & 0.15 & 4.9 & 4.8 \\
\hline Li1- & $1.908(1)$ & $-*$ & $-*$ & 0.03 & 0.05 & 0.18 & 0.17 & 5.7 & 5.7 \\
\hline \multicolumn{10}{|l|}{$\mathrm{O} 1 \mathrm{~W}$} \\
\hline $\mathrm{S} 1-\mathrm{C} 4$ & $1.7839(8)$ & $-*$ & $-*$ & $0.14(2)$ & $0.08(1)$ & $1.32(1)$ & 1.27 & $-6.1(4)$ & $-5.1(1)$ \\
\hline S1-C5 & $1.7910(9)$ & $-^{*}$ & $-*$ & $0.13(2)$ & 0.07 & $1.31(1)$ & 1.26 & $-5.9(4)$ & $-5.0(1)$ \\
\hline $\mathrm{S} 1-\mathrm{O} 3$ & $1.508(1)$ & $-^{*}$ & $-^{*}$ & $0.19(3)$ & $0.11(1)$ & $1.89(2)$ & 1.77 & $-1.9(18)$ & $3.8(4)$ \\
\hline $\mathrm{C} 4-\mathrm{H} 4 \mathrm{~A}$ & $1.077(8)$ & $0.68(2)$ & $1.00(1)$ & 0.00 & 0.03 & $1.75(1)$ & 2.12 & $-14.5(4)$ & $-25.0(3)$ \\
\hline C4-H4B & $1.078(6)$ & $0.68(2)$ & $0.95(2)$ & 0.01 & 0.01 & $1.75(1)$ & 2.01 & $-14.4(4)$ & $-22.3(3)$ \\
\hline $\mathrm{C} 4-\mathrm{H} 4 \mathrm{C}$ & $1.076(7)$ & $0.69(2)$ & $1.08(1)$ & 0.00 & 0.01 & $1.75(1)$ & 2.17 & $-14.5(4)$ & $-25.9(2)$ \\
\hline C5-H5A & $1.077(9)$ & $0.68(2)$ & $0.93(1)$ & 0.02 & 0.01 & $1.74(1)$ & 2.07 & $-14.4(4)$ & $-24.0(2)$ \\
\hline C5-H5B & $1.077(6)$ & $0.68(2)$ & $0.93(1)$ & 0.01 & 0.01 & $1.75(1)$ & 2.07 & $-14.5(4)$ & $-23.8(2)$ \\
\hline C5-H5C & $1.077(6)$ & $0.69(2)$ & $1.31(2)$ & 0.01 & 0.01 & $1.75(1)$ & 2.20 & $-14.5(4)$ & $-27.3(4)$ \\
\hline $\begin{array}{l}\text { O1W- } \\
\text { H1WA }\end{array}$ & $0.979(6)$ & 0.60 & $0.70(1)$ & 0.02 & 0.02 & $2.41(1)$ & $3.27(2)$ & $-31.3(8)$ & $\begin{array}{l}-97.2(32 \\
)\end{array}$ \\
\hline
\end{tabular}


O1W-

H1WB

$0.980(6) \quad 0.60$

0.76

0.03

0.04

2.41(1) 3.07(1) $-31.1(7)$

$-71.3(24$

Symmetry operators: (i) $-x,-y,-z$, (ii) $1-x, 2-y,-z$, (iii) $1+x, 2-y, 1-z$.

* The empirical approach used to determine bond orders relies on parametrisation, but no parameters for $\mathrm{Li}-\mathrm{O}, \mathrm{S}-\mathrm{O}$ and $\mathrm{S}-\mathrm{C}$ bond orders exists in literature to the best authors' knowledge. 
Table S4 Comparison of bond lengths $(\AA ̊)$ in quinone rings of 2,5-dihydroxyquinonates with various 3,6-substituents. [1,2,4]

\begin{tabular}{|c|c|c|c|c|c|}
\hline Bond & $\mathrm{DHQ}^{2-}$ & $\mathrm{H}_{2} \mathrm{CA}$ & $\mathrm{HCA}^{-\mathrm{a}}$ & $\mathrm{CA}^{2-}$ & $\mathrm{NA}^{2-}$ \\
\hline $\mathrm{C} 1-\mathrm{C} 2$ & $1.5348(7)$ & $1.5083(3)$ & $1.5059(17)$ & $1.5413(2)$ & $1.5539(3)$ \\
\hline $\mathrm{C} 2-\mathrm{C} 3$ & $1.4004(7)$ & $1.3576(3)$ & $1.3577(15)$ & $1.4009(2)$ & $1.4129(2)$ \\
\hline $\mathrm{C} 3-\mathrm{C} 1^{\mathrm{b}}$ & $1.3965(7)$ & $1.4515(3)$ & $1.4551(16)$ & $1.4045(3)$ & $1.4356(3)$ \\
\hline $\mathrm{C} 4-\mathrm{C} 5$ & & & $1.5419(17)$ & & \\
\hline $\mathrm{C} 5-\mathrm{C} 6$ & & & $1.4068(15)$ & & \\
\hline C6-O1 & & & $1.4095(16)$ & & \\
\hline $\mathrm{C} 1-\mathrm{O} 1$ & $1.2655(7)$ & $1.2241(3)$ & $1.2450(14)$ & $1.2467(3)$ & $1.2293(2)$ \\
\hline $\mathrm{C} 2-\mathrm{O} 2$ & $1.2630(7)$ & $1.3117(3)$ & $1.3182(15)$ & $1.2484(3)$ & $1.2466(2)$ \\
\hline $\mathrm{C} 4-\mathrm{O} 3$ & & & $1.2227(14)$ & & \\
\hline $\mathrm{C} 5-\mathrm{O} 4$ & & & $1.2430(14)$ & & \\
\hline $\mathrm{O} 2-\mathrm{H} 2^{\mathrm{c}}$ & & $0.979(2)$ & $1.3182(15)$ & & \\
\hline $\mathrm{C} 3-\mathrm{H} 3^{\mathrm{c}}$ & $1.083(2)$ & & & & \\
\hline $\mathrm{C} 3-\mathrm{N} 1$ & & & & & $1.4414(3)$ \\
\hline $\mathrm{C} 3-\mathrm{Cl1} 1^{\mathrm{b}}$ & & $1.7121(3)$ & $1.7214(18)$ & $1.7368(2)$ & \\
\hline $\mathrm{C} 6-\mathrm{Cl} 2$ & & & $1.7196(18)$ & & \\
\hline
\end{tabular}

a Hydrogenchloranilate atoms are numerated differently in reference [4]. To facilitate comparisons, the correspondence (reference $\rightarrow$ this table) is: $\mathrm{C} 1 \rightarrow \mathrm{C} 2, \mathrm{C} 2 \rightarrow \mathrm{C} 1, \mathrm{C} 4 \rightarrow \mathrm{C} 5$, $\mathrm{C} 5 \rightarrow \mathrm{C} 4, \mathrm{O} 1 \rightarrow \mathrm{O} 2, \mathrm{O} 2 \rightarrow \mathrm{O} 1, \mathrm{O} 3 \rightarrow \mathrm{O} 4, \mathrm{O} 4 \rightarrow \mathrm{O} 3, \mathrm{H} 1 \rightarrow \mathrm{H} 2, \mathrm{Cl} 1 \rightarrow \mathrm{Cl} 2, \mathrm{Cl} 2 \rightarrow \mathrm{Cl} 1$. The $\pi$-system in $\mathrm{HCA}^{-}$consists of atoms: $\mathrm{C} 2, \mathrm{O} 2, \mathrm{C} 3, \mathrm{C} 4, \mathrm{O} 3$, with $\mathrm{Cl} 1$ on its periphery. The non- $\pi$-system consists of atoms: $\mathrm{C} 5, \mathrm{O} 4, \mathrm{C} 6, \mathrm{C} 1, \mathrm{O} 1$, with $\mathrm{H} 1$ and $\mathrm{Cl} 2$ on its periphery.

$\mathrm{b}$ These $\mathrm{C} 1$ and $\mathrm{C} 3$ atoms are not from the asymmetric unit, but from the centrosymmetric equivalent needed to complete the quinone ring. The symmetry operator differs in different structures. $\mathrm{HCA}^{-}$ion is not centrosymmetric; the value in this row refers to the $\mathrm{C} 3-\mathrm{C} 4$ bond.

${ }^{c} \mathrm{O}-\mathrm{H}$ and $\mathrm{C}-\mathrm{H}$ bonds are restrained during refinement to quoted values. 
Table S5 Geometric parameters of hydrogen bonds.

\begin{tabular}{|c|c|c|c|c|c|}
\hline & $D-\mathrm{H} / \AA$ & $\mathrm{H} \cdots A / \AA$ & $D \cdots A / \AA$ & $\underset{\circ}{D-\mathrm{H} \cdots A} /$ & Symm. op. on $A$ \\
\hline O1W-H1WA $\cdots \mathrm{O} 1$ & $0.979(6)$ & $1.766(6)$ & $2.7385(7)$ & $172.0(5)$ & $1-x, 2-y, 1-z$ \\
\hline $\mathrm{O} 1 \mathrm{~W}-\mathrm{H} 1 \mathrm{WB} \cdots \mathrm{O} 2$ & $0.980(6)$ & $1.739(6)$ & $2.7160(7)$ & $174.4(6)$ & $-1 / 2+X, 3 / 2-y,-1 / 2+z$ \\
\hline $\mathrm{C} 3-\mathrm{H} 3 \cdots \mathrm{O} 33^{*}$ & $1.083(8)$ & $2.963(7)$ & $3.838(8)$ & $134.3(8)$ & $1 / 2+x, 3 / 2-y, 1 / 2+z$ \\
\hline $\mathrm{C} 4-\mathrm{H} 4 \mathrm{~A} \cdots \mathrm{O} 1 \mathrm{~W}^{*}$ & $1.077(8)$ & $2.859(8)$ & $3.811(9)$ & $147.5(8)$ & $1 / 2+x, 3 / 2-y,-1 / 2+z$ \\
\hline $\mathrm{C} 4-\mathrm{H} 4 \mathrm{~B} \cdots \mathrm{O} 3$ & $1.078(6)$ & $2.465(6)$ & $3.535(1)$ & $172.0(8)$ & $1-x, 2-y,-z$ \\
\hline $\mathrm{C} 4-\mathrm{H} 4 \mathrm{C} \cdots \mathrm{O} 1$ & $1.076(7)$ & $2.674(6)$ & $3.630(1)$ & $147.7(7)$ & $-1 / 2+x, 3 / 2-y,-1 / 2+z$ \\
\hline C5-H5A $\cdots \mathrm{O} 1 \mathrm{~W}$ & $1.077(9)$ & $2.649(8)$ & $3.663(1)$ & $156.7(6)$ & $1 / 2+X, 3 / 2-y,-1 / 2+z$ \\
\hline 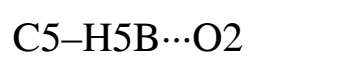 & $1.077(5)$ & $2.524(9)$ & $3.318(1)$ & $129.8(8)$ & $1-x, 1-y, 1-z$ \\
\hline C5-H5C $\cdots \mathrm{O} 3$ & $1.077(7)$ & $2.617(10)$ & $3.475(1)$ & $131.1(8)$ & $3 / 2-x,-1 / 2+y, 1 / 2-z$ \\
\hline
\end{tabular}

* Normally, these contacts would not appear in this table, as the $\mathrm{H} \cdots \mathrm{O}$ distance is too large to justify their inclusion as H-bonds. However, they complement the critical point information from Table 4. 


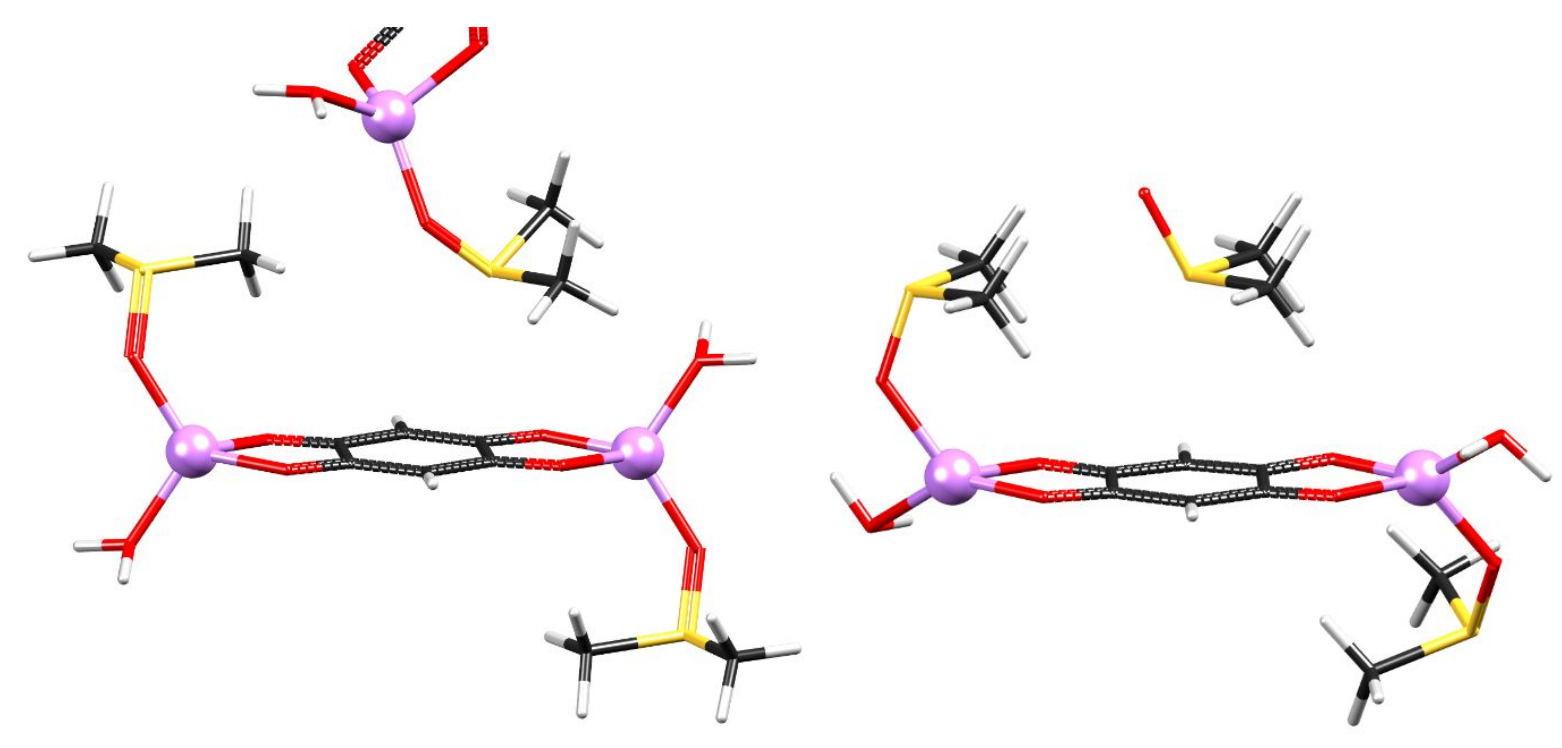

Figure S9 Comparison of experimental (left) and optimized (right) contact between DMSO and DHQ dianion.

Table S6 Geometric parameters of contact between DMSO and DHQ dianion for experimental and optimized structures.

\begin{tabular}{lll}
\hline$d / \AA$ & $\exp$ & theo \\
\hline $\mathrm{S} 1 \cdots$ centroid & 3.461 & 3.400 \\
$\mathrm{~S} 1 \cdots \mathrm{C} 1$ & $3.4046(7)$ & 3.646 \\
$\mathrm{~S} 1 \cdots \mathrm{C} 2$ & $3.2481(7)$ & 3.697 \\
$\mathrm{~S} 1 \cdots \mathrm{C} 3$ & $3.8731(7)$ & 3.687 \\
\hline
\end{tabular}

\section{S5 References}

[1] V. Vuković, K. Molčanov, C. Jelsch, E. Wenger, A. Krawczuk, M. Jurić, L. Androš Dubraja, B. Kojić-Prodić, Cryst. Growth Des., 19 (2019), 2802-2810.

[2] K. Molčanov, C. Jelsch, E. Wenger, J. Stare, A. Ø. Madsen, B. Kojić-Prodić, CrystEngComm, 19 (2017), 3898-3901.

[3] S. Domagała, B. Fournier, D. Liebschner, B. Guillot \& C. Jelsch, Acta Crystallogr. A68 (2012) 337. 
[4] K. Molčanov, J. Stare, B. Kojić-Prodić, C. Lecomte, S. Dahaoui, C. Jelsch, E. Wenger, A. Šantić \& B. Zarychta, CrystEngComm 17 (2015) 8645. 